Original Article

\title{
Does transcutaneous electrical nerve stimulation (TENS) simultaneously combined with local heat and cold applications enhance pain relief compared with TENS alone in patients with knee osteoarthritis?
}

\author{
Takaya Maeda, RPT, MS ${ }^{1,2)^{*}}$, Hideki Yoshida, RPT, PhD²), Tomoyuki Sasaki, MD, PhD ${ }^{1)}$, \\ Atsushi ODA, RPT, PhD 2 ) \\ 1) Department of Rehabilitation, Hirosaki Memorial Hospital: 59-1 Sakaizeki Nishida, Hirosaki-city, \\ Aomori 036-8067, Japan \\ 2) Department of Comprehensive Rehabilitation Science, Hirosaki University Graduate School of \\ Health Sciences, Japan
}

\begin{abstract}
Purpose] The purpose of this study was to investigate whether transcutaneous electrical nerve stimulation simultaneously combined with local heat and cold applications enhances pain relief compared with transcutaneous electrical nerve stimulation alone in patients with knee osteoarthritis. [Subjects and Methods] Fourty-five patients with knee osteoarthritis participated in this study. They were randomly assigned to the following three interventions: transcutaneous electrical nerve stimulation simultaneously combined with local heat using a hot pack; combined with local cold using a cold pack; and transcutaneous electrical nerve stimulation alone. In each intervention, the knee pain level during walking and standing up from a chair, as well as dynamic balance and gait ability were evaluated immediately before and after a single intervention using the visual analogue scale and the timed up \& go test, respectively. [Results] A significant improvement in dynamic balance and gait ability was only observed immediately after transcutaneous electrical nerve stimulation simultaneously combined with local heat application, although the degree of pain relief during standing and walking were comparable among the three interventions. [Conclusion] These results suggest that transcutaneous electrical nerve stimulation simultaneously combined with local heat application can immediately improve not only knee pain during standing and walking but also dynamic balance and gait ability in patients with knee osteoarthritis.

Key words: Knee osteoarthritis, Transcutaneous electrical nerve stimulation, Thermal modality
\end{abstract}

(This article was submitted May 29, 2017, and was accepted Jul. 22, 2017)

\section{INTRODUCTION}

Knee osteoarthritis (OA) is the most common type of OA and often leads to pain, swelling, decreased joint movements, stiffness, or the formation of bone spurs ${ }^{1}$. According to the OA Research Society International guidelines for the management of hip and knee OA, optimal management of OA requires a combination of nonpharmacological and pharmacological modalities, transcutaneous electrical nerve stimulation (TENS) and certain thermal modalities (i.e., local heat and cold applications) may be effective for relieving symptoms of knee $\mathrm{OA}^{2,3)}$.

TENS is a method of electrical stimulation which primarily aims to provide a degree of symptomatic pain relief by excit-

*Corresponding author. Takaya Maeda (E-mail: takamaru3250@live.jp)

(C2017 The Society of Physical Therapy Science. Published by IPEC Inc.

(c) (1) $\odot$ This is an open-access article distributed under the terms of the Creative Commons Attribution Non-Commercial No DerivacC. 
ing sensory nerves, which stimulate either the pain-gate mechanism and/or the opioid system ${ }^{4}$ ). Several systematic reviews have shown that TENS is one of the recommended treatments for pain relief in some patients with knee $\mathrm{OA}^{5-7}$ ). However, a previous study showed that there were no significant differences in performance measures between active TENS and placebo TENS $^{8)}$. Furthermore, a recent systematic review concluded that TENS is not effective for knee OA pain ${ }^{9)}$. Therefore, a consensus regarding the effect of TENS on knee OA pain has not yet been reached.

In addition to TENS, local heat and cold applications are also used widely for patients with knee OA. When therapeutic heat is used, the stimuli activate the spinal gating mechanism (i.e., the gate control theory of pain) ${ }^{10,11)}$. Local heat has also been shown to elevate the pain threshold ${ }^{10,12)}$. On the other hand, local cold is also commonly used to decrease pain. Analgesia is a direct effect of therapeutic cold ${ }^{10)}$. Possible analgesic mechanisms in local cold are partially similar to those in local heat ${ }^{10)}$. However, a systematic review concluded that local heat and cold applications had no beneficial effect on edema or pain in patient with $\mathrm{OA}^{13}$. Therefore, evidence of the effect of local heat and cold on knee OA has not yet been established. In the meantime, a previous study reported that a combined treatment of TENS and therapeutic cold, which consists of a TENS treatment with an ice bag secured over the TENS electrodes, is effective in treating the pain and muscle spasms that occur with delayed-onset muscle soreness ${ }^{14}$ ). The finding suggests that compared with TENS alone, TENS simultaneously combined with the thermal modalities (at least local cold) may provide a synergistic analgesic effect induced by activation of several pain inhibitory mechanisms. However, little is known regarding whether TENS simultaneously combined with local heat or cold demonstrates more effective pain relief compared with TENS alone in patients with knee OA.

The present study thus aimed to investigate whether TENS simultaneously combined with local heat or cold enhances pain relief compared with TENS alone in patients with knee OA.

\section{SUBJECTS AND METHODS}

Fourty-five patients with medial component knee OA were recruited for this study. Participants had a mean age of $66.9 \pm$ 8.48 years, height of $153 \pm 7.56 \mathrm{~cm}$, weight of $63.1 \pm 13.9 \mathrm{~kg}$, and body mass index of $26.8 \pm 4.94 \mathrm{~kg} / \mathrm{m}^{2}$. Only patients who experienced knee pain during walking and standing up from a chair at a comfortable speed were finally selected. Exclusion criteria were as follows: history of serious peripheral circulatory disturbance, history of heart or respiratory problems, history of central or peripheral neurological disorders, and history of dementia. All patients provided informed consent to participate in this study. This study was conducted in accordance with the Declaration of Helsinki and was approved by the Hirosaki Memorial Hospital Research Ethics Committee (H27-01).

Participants were randomly divided into three groups by lottery in which the following were performed for treatment of knee pain: (1) TENS only (control group); (2) TENS simultaneously combined with local heat application (TENS+LH group); (3) TENS simultaneously combined with local cold application (TENS+LC group). The characteristics of the subjects in each group is presented in Table 1. In all three groups, TENS was performed using a portable electrical stimulation device (ESPURGE, ITO Co. Ltd., Tokyo, Japan). Electrical stimulation was delivered using two disposable, $50 \times 50 \mathrm{~mm}$, self-adhesive surface electrodes (PALS; Axelgard Manufacturing Co. Ltd., Fallbrook, USA). The electrodes were placed so as to sandwich the medial side of the knee that hurt when walking and rising from a chair. The spinal segments that innervate the sensation of both the periosteum and joint capsule of the medial side of the knee are L3 or L4, according to the human sclerotome map ${ }^{15)}$. Therefore, the two electrodes for TENS were placed on the skin of the thigh and lower leg matching L3 and L4 dermatome maps in order to obtain sufficient pain relief according to the principle of spinal sensory gating. In all three groups, TENS parameters were set as follows: monophasic square waveform, strong (muscle contraction level) but comfortable intensity, 100 pps frequency, 200- $\mu$ s pulse width, and 20-min stimulation duration. In the TENS+LH group, a dry hot pack (HYDROPACMEL PX-151, OG GIKEN, Co. Ltd., Tokyo, Japan) with a surface temperature of approximately $40^{\circ} \mathrm{C}$ was placed on the two electrodes for the duration of the TENS session. In the TENS+LC group, a cold pack (MOIST HEAT-PACK, Duro-Med Industries, Inc., GA, USA) with a surface temperature of approximately $10^{\circ} \mathrm{C}$ was covered with a cotton towel and placed on the two electrodes for the duration of the TENS session. In this study, one TENS session was conducted for each participant and its immediate effect was examined using the evaluation indices described below.

The Visual Analogue Scale (VAS) was used to evaluate the level of knee pain during both walking (w-VAS) and standingup from a chair (s-VAS) at a comfortable speed. The VAS was a 100-mm-long horizontal line anchored by word descriptors at each end ${ }^{16)}$. The left side of the scale $(0 \mathrm{~mm})$ read "no pain", and the right side of the scale $(100 \mathrm{~mm})$ read "worst pain imaginable". Each participant drew a vertical mark on the line at the point that best represented their perceived pain. Both the w-VAS and s-VAS were evaluated immediately before and after the TENS intervention in each group. The timed up and go test (TUG) was performed to evaluate the improvement in dynamic balance and gait ability, reflecting TENS related pain relief ${ }^{17)}$. In this study, the TUG test was performed by first asking the participant to wait, sitting with their back against a chair without armrest (seat height, $43 \mathrm{~cm}$; depth, $41 \mathrm{~cm}$; width, $42 \mathrm{~cm}$ ) that had been placed at the end of a marked 3-m walkway. The participant was then told that when the instructor said "go," they should stand up, walk at a comfortable speed past the 3-m mark, turn around, walk back, and sit down in the chair. The participant was allowed to use assistive and/or orthotic devices if necessary. We used a stopwatch to measure the time required to complete TUG and recorded it in seconds. Measurements of the time required for TUG were performed immediately before and after the TENS intervention in each group. At each measurement time point, TUG was performed twice, and the participant's higher speed was used for statistical 
Table 1. Characteristics of the subjects in each group

\begin{tabular}{lccc}
\hline & TENS+HS group & TENS+HS group & Control group \\
\hline Number of participants & 15 & 15 & 15 \\
Age (years, mean \pm SD) & $64.5 \pm 9.7$ & $66.9 \pm 8.1$ & $69.3 \pm 6.6$ \\
Kellgren-Laurence grade & I:3,II:5,III:2,IV:5 & I:3,II:6,III:3,IV:3 & I:2,II:6,III:2,IV:5 \\
\hline
\end{tabular}

analysis.

In statistical analyses, after changes in the raw w-VAS ( $\Delta \mathrm{w}$-VAS), s-VAS ( $\Delta \mathrm{s}-\mathrm{VAS})$, and TUG $(\Delta T \mathrm{TUG})$ values immediately before and after the TENS intervention in each group were calculated, a one-way analysis of variance was used to compare the $\Delta \mathrm{w}$-VAS, $\Delta \mathrm{s}-\mathrm{VAS}$, and $\triangle \mathrm{TUG}$ values among the three groups. A paired t-test was used to compare the raw w-VAS, s-VAS, and TUG values immediately before and after the TENS intervention within each group. The threshold for significance was set at $\mathrm{p}<0.05$. All statistical analyses were performed using $\mathrm{R}$ version 2.8.1.

\section{RESULTS}

No side effects caused by TENS or local heat and cold were noted. Table 2 presents means and standard deviations (SD) of the raw w-VAS immediately before and after the TENS intervention and those of the $\triangle \mathrm{w}$-VAS value in each group. There were no significant differences in the $\triangle \mathrm{w}$-VAS values among the three groups. In all the groups, however, the raw w-VAS value immediately after the TENS intervention was significantly decreased compared with that immediately before it.

Table 3 presents means and SD of the raw s-VAS values immediately before and after the TENS intervention and those of the $\triangle \mathrm{s}$-VAS value in each group. There were no significant differences in the $\Delta \mathrm{s}$-VAS values among the three groups. In all the groups, however, the raw s-VAS value immediately after the TENS intervention was significantly decreased compared with that immediately before it.

Finally, Table 4 presents means and SD of the raw TUG values immediately before and after the TENS intervention and those of the $\triangle T$ TUG value in each group. There were no significant differences in the $\triangle T U G$ values among the three groups. In addition, there were no significant differences in the raw TUG values immediately before and after the TENS intervention in the control and TENS+LC groups. In the TENS+LH group, however, the raw TUG value immediately after the TENS intervention was significantly decreased compared with that immediately before it.

\section{DISCUSSION}

In the present study, we investigated whether TENS simultaneously combined with local heat or cold applications enhances pain relief compared with TENS alone in patients with knee OA. Our findings indicated that in the TENS + LH, TENS + LC, and control groups, both the raw w-VAS and s-VAS values immediately after the TENS intervention were significantly decreased compared with those immediately before it. These results indicate that apparent relief of knee pain during both standing and walking can be obtained not only via TENS simultaneously combined with local heat or cold but also via TENS alone in patients with knee OA. On the other hand, there were no significant differences in both the $\Delta \mathrm{w}$-VAS and $\Delta \mathrm{s}$-VAS values among the three groups. This finding suggest that TENS simultaneously combined with local heat or cold does not enhance relief of knee pain during standing or walking in patients with knee OA. In other words, this type of knee pain can be sufficiently suppressed even with TENS alone. TENS performed at a frequency of $100 \mathrm{pps}$, as adopted in this study, can selectively activate large-diameter A $\beta$ fibers (touch related) without concurrently activating small-diameter A $\delta$ and $\mathrm{C}$ fibers (pain related) ${ }^{4}, 11$. TENS-induced large-diameter afferent activity inhibits ongoing transmission of pain information in the spinal cord (i.e., the gate control theory of pain) ${ }^{4,11)}$. This pain relief is achieved during stimulation, with a limited "carry over" effect ${ }^{4}$. In the meantime, TENS implemented at about 100 pps releases dynorphin, one of the endogenous opioids, with the analgesic effect mediated via kappa opioid receptors $\left.{ }^{4}, 11\right)$. In general, the onset of pain relief mediated via opioid receptors may be slower than that of the pain-gate mechanism, as it takes time to increase opioid level via TENS. However, once a sufficient level of opioids has been released, it will continue to work strongly for at least 30-60 minutes, even after the cessation of the stimulation ${ }^{4}{ }^{18)}$. As with TENS, application of local heat and cold can provide pain relief. A proposed pain-relief mechanism common to both local heat and cold includes the gate control theory of pain ${ }^{10}$. Apart from this, an increase in the current perception threshold of sensory nerve fibers associated with local heat ${ }^{10)}$, and decreased nerve conduction velocity associated with local cold ${ }^{10)}$, are also involved in pain relief. Among these, the latter two pain-relief mechanisms can induce a carry-over effect lasting for several tens of minutes ${ }^{10,12)}$. In previous studies, however, the degree of the carry-over effect induced by local heat and cold was not fully examined. Results of the current study may indicate that the carry-over effect on pain relief caused by local heat and cold is weaker than that caused by TENS. Therefore, TENS simultaneously combined with local heat or cold does not provide a synergistic analgesic interaction compared with TENS alone.

In addition, the raw TUG value immediately after the TENS intervention was significantly decreased compared with 
Table 2. Raw w-VAS values immediately before and after TENS intervention and $\Delta \mathrm{w}$-VAS values in each group $[\mathrm{mm}]$

\begin{tabular}{lccc}
\hline & $\begin{array}{c}\text { Immediately before } \\
\text { TENS intervention } \\
(\text { Mean } \pm \text { SD) }\end{array}$ & $\begin{array}{c}\text { Immediately after } \\
\text { TENS intervention } \\
(\text { Mean } \pm \text { SD) }\end{array}$ & $\begin{array}{c}\Delta \mathrm{w}-\mathrm{VAS} \\
(\text { Mean } \pm \text { SD) }\end{array}$ \\
\hline TENS+HS group & $36.07 \pm 27.08$ & $24.20 \pm 21.44^{*}$ & $-11.87 \pm 13.95$ \\
TENS+CS group & $46.93 \pm 27.65$ & $28.07 \pm 25.67^{*}$ & $-18.87 \pm 19.25$ \\
Control group & $38.93 \pm 25.93$ & $26.47 \pm 23.75^{*}$ & $-12.47 \pm 13.46$ \\
\hline
\end{tabular}

$* \mathrm{p}<0.05$ : comparison immediately before and after TENS intervention in each group

Table 3. Raw s-VAS values immediately before and after TENS intervention and $\triangle \mathrm{s}-\mathrm{VAS}$ values in each group $[\mathrm{mm}]$

\begin{tabular}{lccc}
\hline & $\begin{array}{c}\text { Immediately before } \\
\text { TENS intervention } \\
(\text { Mean } \pm \text { SD) }\end{array}$ & $\begin{array}{c}\text { Immediately after } \\
\text { TENS intervention } \\
(\text { Mean } \pm \text { SD) }\end{array}$ & $\begin{array}{c}\Delta \mathrm{s} \text {-VAS } \\
(\text { Mean } \pm \text { SD) }\end{array}$ \\
\hline TENS+HS group & $29.73 \pm 29.52$ & $17.87 \pm 21.84^{*}$ & $-11.87 \pm 19.50$ \\
TENS+CS group & $34.20 \pm 26.02$ & $18.20 \pm 24.08^{*}$ & $-16.00 \pm 23.06$ \\
Control group & $32.87 \pm 27.59$ & $22.40 \pm 22.13^{*}$ & $-10.47 \pm 9.02$ \\
\hline
\end{tabular}

${ }^{*} \mathrm{p}<0.05$ : comparison immediately before and after TENS intervention in each group

Table 4. Raw TUG values immediately before and after TENS intervention and $\triangle \mathrm{TUG}$ values in each group [sec]

\begin{tabular}{lccc}
\hline & $\begin{array}{c}\text { Immediately before } \\
\text { TENS intervention } \\
(\text { Mean } \pm \mathrm{SD})\end{array}$ & $\begin{array}{c}\text { Immediately after } \\
\text { TENS intervention } \\
(\text { Mean } \pm \mathrm{SD})\end{array}$ & $\begin{array}{c}\Delta \mathrm{TUG} \\
\text { (Mean } \pm \mathrm{SD})\end{array}$ \\
\hline TENS+HS group & $11.91 \pm 5.74$ & $10.87 \pm 4.80^{*}$ & $-1.04 \pm 1.58$ \\
TENS+CS group & $9.52 \pm 2.51$ & $9.30 \pm 2.44$ & $-0.22 \pm 0.62$ \\
Control group & $9.69 \pm 2.52$ & $9.55 \pm 2.46$ & $-0.14 \pm 0.93$ \\
\hline * $<$ 0.05: comparison immediately before and after TENS intervention in each group
\end{tabular}

that immediately before it in the TENS+LH group only, although there were no significant differences in the $\triangle T$ TU values among the three groups. Considering the abovementioned results on both the w-VAS and s-VAS, the TUG results suggest that only the TENS simultaneously combined with local heat can improve dynamic balance and gait ability. Nevertheless, the pain-relief effect of TENS simultaneously combined with local heat or cold is equivalent to that of TENS alone in patients with knee OA. As an explanation for this, the influence of "passive warm up" due to heat stimulation is suggested. Bishop ${ }^{19)}$ indicated that passive warm up could increase the temperature of the skeletal muscle, resulting in an improvement in dynamic force. In the TENS+LH group, local heat around the knee joint for 20 minutes was administered using a hot pack. There is no doubt that the local heat corresponds to passive warm up. In addition, Oosterveld and colleagues ${ }^{20)}$ pointed out that a hot pack, which is superficial heating stimuli, can alter intra-articular temperature of the knee joint. If so, it is likely that the hot pack used in this study could also increase the temperature of the skeletal muscles around the knee joint, resulting in an improvement in dynamic balance and gait ability. From a clinical standpoint, the use of TENS simultaneously combined with local heat will be recommended when therapeutic exercises such as gait exercise are performed immediately after pain-relief by using TENS.

The present study has some limitations. First, it suggested the possibility that the carry-over effect on pain relief caused by local heat and cold was weaker than that caused by TENS. Therefore, TENS simultaneously combined with local heat or cold did not provide a synergistic analgesic interaction compared with TENS alone. However, the degree of the carry-over effect on pain relief caused by local heat and cold has not been fully clarified, and it is necessary to elucidate the degree of this effect to provide evidence for the abovementioned possibility. In addition, a clinically interesting finding of our study was that only TENS simultaneously combined with local heat was able to provide immediate improvement in dynamic balance and gait ability in patients with knee OA. This finding may indicate that even from a long-term perspective, TENS simultaneously combined with local heat can provide a significant improvement in dynamic balance and gait ability compared with TENS alone in patients with knee OA. Future studies should investigate the relationship between TENS simultaneously combined with local heat and the long-term improvement in dynamic balance and gait ability in patients with knee OA. 


\section{REFERENCES}

1) Parmet S, Lynm C, Glass RM: JAMA patient page. Osteoarthritis of the knee. JAMA, 2003, 289: 1068. [Medline] [CrossRef]

2) Zhang W, Moskowitz RW, Nuki G, et al.: OARSI recommendations for the management of hip and knee osteoarthritis, part II: OARSI evidence-based, expert consensus guidelines. Osteoarthritis Cartilage, 2008, 16: 137-162. [Medline] [CrossRef]

3) McAlindon TE, Bannuru RR, Sullivan MC, et al.: OARSI guidelines for the non-surgical management of knee osteoarthritis. Osteoarthritis Cartilage, 2014, 22: 363-388. [Medline] [CrossRef]

4) Watson T: Electrotherapy: evidence-based practice. Philadelphia: Churchill Livingstone, 2008.

5) Osiri M, Welch V, Brosseau L, et al.: Transcutaneous electrical nerve stimulation for knee osteoarthritis. Cochrane Database Syst Rev, 2000, 4: CD002823 10.1002/14651858.CD002823. [Medline]

6) Brosseau L, Yonge K, Marchand S, et al.: Efficacy of transcutaneous electrical nerve stimulation for osteoarthritis of the lower extremities: a meta-analysis. Phys Ther Rev, 2004, 9: 213-233. [CrossRef]

7) Bjordal JM, Johnson MI, Lopes-Martins RA, et al.: Short-term efficacy of physical interventions in osteoarthritic knee pain. A systematic review and metaanalysis of randomised placebo-controlled trials. BMC Musculoskelet Disord, 2007, 8: 51. [Medline] [CrossRef]

8) Vance CG, Rakel BA, Blodgett NP, et al.: Effects of transcutaneous electrical nerve stimulation on pain, pain sensitivity, and function in people with knee osteoarthritis: a randomized controlled trial. Phys Ther, 2012, 92: 898-910. [Medline] [CrossRef]

9) Rutjes AW, Nüesch E, Sterchi R, et al.: Transcutaneous electrostimulation for osteoarthritis of the knee. Cochrane Database Syst Rev, 2009 , 4: CD002823 10.1002/14651858.CD002823.pub2. [Medline]

10) Cameron MH: Physical agents: from research to practice. St. Louis: Sunders, 2008.

11) Johnson MI: Transcutaneous electrical nerve stimulation (TENS): research to support clinical practice. Oxford: Oxford University Press, 2014.

12) Benson TB, Copp EP: The effects of therapeutic forms of heat and ice on the pain threshold of the normal shoulder. Rheumatol Rehabil, 1974, 13: 101-104. [Medline] [CrossRef]

13) Brosseau L, Yonge KA, Robinson V, et al.: Thermotherapy for treatment of osteoarthritis. Cochrane Database Syst Rev, 2003, 4: CD004522 10.1002/14651858. CD004522. [Medline]

14) Denegar CR, Perrin DH: Effect of transcutaneous electrical nerve stimulation, cold, and a combination treatment on pain, decreased range of motion, and strength loss associated with delayed onset muscle soreness. J Athl Train, 1992, 27: 200-206. [Medline]

15) McCredie J, Willert HG: Longitudinal limb deficiencies and the sclerotomes. An analysis of 378 dysmelic malformations induced by thalidomide. J Bone Joint Surg Br, 1999, 81: 9-23. [Medline] [CrossRef]

16) Huskisson EC: Measurement of pain. Lancet, 1974, 2: 1127-1131. [Medline] [CrossRef]

17) Podsiadlo D, Richardson S: The timed "Up \& Go": a test of basic functional mobility for frail elderly persons. J Am Geriatr Soc, 1991, 39: 142-148. [Medline] [CrossRef]

18) Chesterton LS, Foster NE, Wright CC, et al.: Effects of TENS frequency, intensity and stimulation site parameter manipulation on pressure pain thresholds in healthy human subjects. Pain, 2003, 106: 73-80. [Medline] [CrossRef]

19) Bishop D: Warm up I: potential mechanisms and the effects of passive warm up on exercise performance. Sports Med, 2003, 33: 439-454. [Medline] [CrossRef]

20) Oosterveld FG, Rasker JJ, Jacobs JW, et al.: The effect of local heat and cold therapy on the intraarticular and skin surface temperature of the knee. Arthritis Rheum, 1992, 35: 146-151. [Medline] [CrossRef] 